\title{
Microreview
}

\section{Biological systems of the host cell involved in Agrobacterium infection}

Vitaly Citovsky, ${ }^{1}$ Stanislav V. Kozlovsky, ${ }^{1}$ Benoît Lacroix, ${ }^{1}$ Adi Zaltsman, ${ }^{1}$ Mery Dafny-Yelin, ${ }^{2}$ Shachi Vyas, ${ }^{2}$ Andriy Tovkach ${ }^{2}$ and Tzvi Tzfira ${ }^{2 *}$ ${ }^{1}$ Department of Biochemistry and Cell Biology, State University of New York, Stony Brook, NY 11794, USA. ${ }^{2}$ Department of Molecular, Cellular and Developmental Biology, The University of Michigan, Ann Arbor, MI 48109, USA.

\section{Summary}

Genetic transformation of plants by Agrobacterium, which in nature causes neoplastic growths, represents the only known case of trans-kingdom DNA transfer. Furthermore, under laboratory conditions, Agrobacterium can also transform a wide range of other eukaryotic species, from fungi to sea urchins to human cells. How can the Agrobacterium virulence machinery function in such a variety of evolutionarily distant and diverse species? The answer to this question lies in the ability of Agrobacterium to hijack fundamental cellular processes which are shared by most eukaryotic organisms. Our knowledge of these host cellular functions is critical for understanding the molecular mechanisms that underlie genetic transformation of eukaryotic cells. This review outlines the bacterial virulence machinery and provides a detailed discussion of seven major biological systems of the host cell-cell surface receptor arrays, cellular motors, nuclear import, chromatin targeting, targeted proteolysis, DNA repair, and plant immunity - thought to participate in the Agrobacteriummediated genetic transformation.

\section{Introduction}

The ability of Agrobacterium to genetically transform a wide variety of plant species has earned it a place of honour in basic plant research and modern plant

Received 12 June, 2006; accepted 27 July, 2006. *For correspondence. E-mail ttzfira@ @umich.edu; Tel. (+1) 734764 3265; Fax (+1) 7347640884 . biotechnology. The transformation results from the production of a single-stranded copy (T-strand) of transferred DNA (T-DNA) molecule by the bacterial virulence machinery, its transfer into the host cell followed by integration into the host genome (for recent reviews, see Gelvin, 2003; McCullen and Binns, 2006). While wildtype Agrobacterium species are known as the causative agents of the 'crown gall' disease in a rather limited number of economically important plant species (e.g. Burr et al., 1998), recombinant Agrobacterium strains are the tool-of-choice for production of genetically modified plants in a very broad range of species (Gelvin, 2003). Furthermore, Agrobacterium, at least under laboratory conditions, can transform other eukaryotic species, ranging from fungi to human cells (reviewed in Lacroix et al., 2006a), which holds great promise for the future of biotechnology of non-plant species. This remarkably wide host range of Agrobacterium, which is in contrast to the relatively narrow host range of many other bacterial pathogens that are typically limited to specific species or genera - raises a question of how the Agrobacterium virulence machinery can function in evolutionarily distant and diverse species, crossing the interkingdom boundaries. The answer most likely lies in the ability of Agrobacterium to hijack fundamental cellular processes which are shared by organisms of different kingdoms. Thus, the Agrobacterium-mediated genetic transformation process relies both on the activity of the bacterial virulence proteins which are required for the early stages of the transformation process (e.g. host recognition and attachment, and T-strand production, Fig. 1), and on the activity of diverse host cellular proteins and systems which are required in the later stages of the transformation process (e.g. nuclear import, integration and expression of the T-DNA, Fig. 1). Here, we summarize the bacterial virulence machinery and then discuss in detail seven major biological systems of the host cell that have been implicated in the Agrobacterium-mediated genetic transformation. Our knowledge of these basic cellular functions, which is critical for understanding the molecular mechanisms that underlie genetic transformation of eukaryotic cells, is enhanced using Agrobacterium as a unique and powerful experimental tool. 


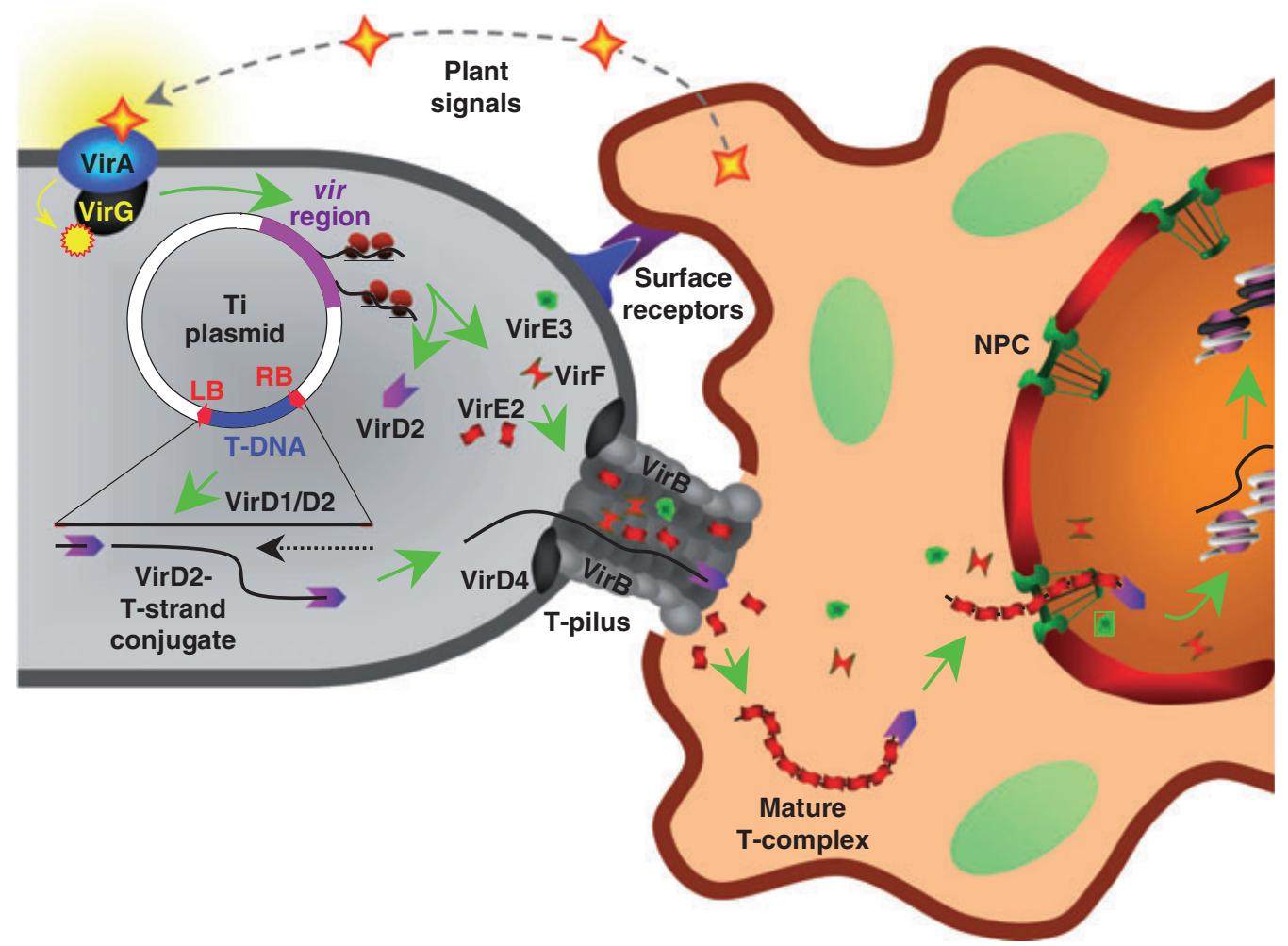

Fig. 1. Summary of major molecular events and structures within the Agrobacterium cell that generate the Vir protein machinery and T-strands which then are transported into the plant cell, enter its nucleus and integrate into the genome. The transformation process begins with recognition of plant signals by the bacterial VirA/VirG sensory system, followed by activation of the vir loci and attachment of the bacterium to the host cell. The T-strand is excised from the T-DNA region by VirD2/VirD1 and exported, in cis with a covalently attached VirD2 molecule and in trans with several other Vir proteins, into the plant cell cytoplasm via a VirB/D4 type IV secretion system. Inside the host cell, the VirD2-T-strand conjugate is packaged by numerous molecules of VirE2 to form a mature T-complex. For in-depth discussion on the T-complex transport and nuclear import, and T-DNA integration, see text.

\section{The bacterial virulence machinery of genetic transformation: a brief overview}

The Agrobacterium-mediated genetic transformation is a multistep process which begins with recognition and sensing of a wounded host cell by a virulent Agrobacterium and ends with the expression of the Agrobacterium's T-DNA integrated in the transformed cell's genome. Agrobacterium deploys a large number of proteins and uses several molecular machines to initiate and execute the early steps of the transformation process, as illustrated in Fig. 1 and as has been previously reviewed (e.g. Gelvin, 2003; Christie et al., 2005; McCullen and Binns, 2006). Briefly, proteins encoded by the bacterial chromosomal virulence (chv) and tumour-inducing plasmid virulence genes ( vir) mediate recognition of and attachment to the host cell, production of a mobile T-strand-protein complex (T-complex) and its export into the host cell (Fig. 1). Once inside the host cell cytoplasm, several Vir proteins and host factors (see below) act together to deliver the T-complex into the host cell nucleus and integrate it into the host cell genome. For detailed discussion of the roles of the Vir proteins in the transformation process, the reader is referred to the recent reviews (e.g. Gelvin, 2003; Christie et al., 2005; McCullen and Binns, 2006).

\section{Plant cell surface receptors and Agrobacterium attachment}

While Agrobacterium attachment to the host cell is an absolute prerequisite for transformation (reviewed in McCullen and Binns, 2006), very little is known about the nature and function of the factors that Agrobacterium utilizes as specific receptors on the host cell surface and/or cell wall. Among these putative receptors and host proteins (Fig. 2) are a vitronectin-like protein (Wagner and Matthysse, 1992), a rhicadhesin-binding protein (Swart et al., 1994), a cellulose synthase-like gene (Zhu et al., 2003a) and several VirB2-interacting proteins (Hwang and Gelvin, 2004). Vitronectins are family of proteins utilized as specific receptors by different pathogenic bacteria in mammalian cells (e.g. Paulsson and Wadstrom, 1990). 


\section{Cell surface receptors}

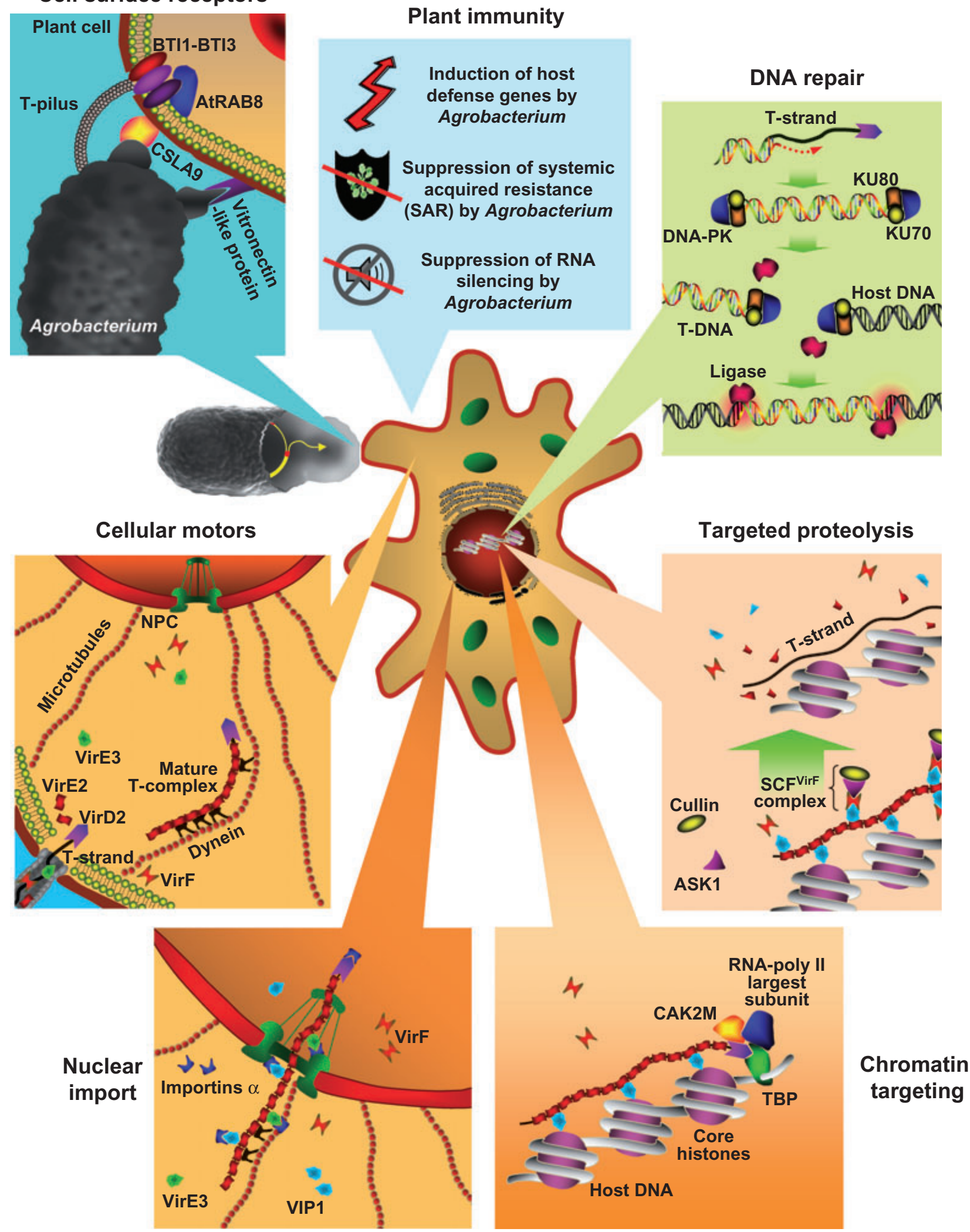

Fig. 2. Summary of major biological systems of the host cell that are involved in the Agrobacterium-mediate genetic transformation. Main molecular events associated with each biological system are depicted. For further details, see text. 
Because attachment of Agrobacterium cells to plant tissues could be inhibited by human vitronectin or antivitronectin antibodies and because Agrobacterium mutants, which are defective in their attachment ability to plant cells, showed reduced binding to vitronectin, plant vitronectin-like molecules have been suggested to play a role in Agrobacterium attachment to its host cells (Wagner and Matthysse, 1992); to date, however, no additional progress has been made in the studies of this putative vitronectin-like plant receptor for Agrobacterium.

In addition to vitronectin, another cell attachmentrelated factor has been postulated to participate in Agrobacterium binding to plant cells. This glycoprotein, isolated from cell walls of pea roots, acted as a receptor for rhicadhesin, an adhesion protein encoded by Agrobacterium and a related phytobacterium Rhizobium; similar to many cell adhesion proteins, including vitronectin, the rhicadhesin receptor contained a conserved arginineglycine-aspartic acid cell attachment motif (Swart et al., 1994).

Another host factor, a cellulose synthase-like protein CSLA9, potentially involved in Agrobacterium attachment, was identified by screening Arabidopsis mutants for resistance to Agrobacterium transformation. Disruption of the CSLA9 gene in Arabidopsis plants resulted in a limited reduction in Agrobacterium attachment to inoculated roots (Zhu et al., 2003a). The CSLA9 promoter driving a GUS reporter gene was active in the root elongation zone (Zhu et al., 2003a), an area previously shown to be most susceptible to Agrobacterium infection (Yi et al., 2002). Thus, while the precise function of the CSLA9 protein in the transformation process remains unknown, its expression pattern - as well as that of $\mathrm{H} 2 \mathrm{~A}$, an Arabidopsis core histone involved T-DNA integration (see below and $\mathrm{Yi}$ et al., 2002) - suggests that Agrobacterium preferentially infects host cells and tissues at specific developmental stages. This notion is lent additional support by the observations that entry into the S-phase of the cell cycle was absolutely essential for the Agrobacterium-mediated stable genetic transformation of synchronized petunia cell populations (Villemont et al., 1997).

Finally, several Arabidopsis proteins have been identified (Hwang and Gelvin, 2004) that interact with VirB2, a major component of the bacterium-host cell attachment structure termed T-pilus (Fig. 2) (Lai and Kado, 2000). These proteins include VirB2 interactors (BTIs) BTI1, $\mathrm{BTI} 2$ and BTI3 with unknown functions, and a membraneassociated GTPase, AtRAB8 (Hwang and Gelvin, 2004). BTI1 and AtRAB8 were implicated in the transformation process using antisense and RNA interference transgenic plants which showed reduced susceptibility to Agrobacterium infection. Interestingly, BTI expression was transiently increased after Agrobacterium infection (Hwang and Gelvin, 2004), indicating a positive feedback commu- nication between Agrobacterium and its host cell. Limited understanding of the T-pilus role in the transformation process (Kado, 2000), combined with the complete lack of knowledge of the biological function(s) of the BTI proteins, hinder our ability to develop a mechanical or molecular model for participation of these host cellular factors in the transformation process.

\section{Molecular motors and intracellular movement of the T-complex}

Following its entry in the host cell cytoplasm, the VirD2T-strand conjugate most likely is coated by VirE2, forming the T-complex (reviewed in Lacroix et al., 2006b), which must then travel through the cytoplasm to reach the host cell nucleus. The dense structure of the cytoplasm, which is composed of a mesh of cytoskeletal networks and greatly restricts the Brownian diffusion of macromolecules (Luby-Phelps, 2000), and the very large size of the T-complex (Abu-Arish et al., 2004) suggest an active mechanism for the intracellular transport of the T-complex (Tzfira, 2006). Studies in mammalian cells have shown that many DNA viruses use dynein motor proteins and the host microtubule network as a track system for their cytoplasmic transport towards the nucleus (reviewed in Henry et al., 2006), and the possibility that Agrobacterium has evolved to utilize the similar transport machinery of the host cell for delivery of the T-complex (Fig. 2) is intriguing. Indeed, a recent biophysical study demonstrated active transport of artificial T-complexes along microtubules in a cell-free system (Salman et al., 2005).

This study used single-particle-tracking methods to track movement of fluorescently labelled VirE2-ssDNA (single-stranded DNA) complexes on a reconstituted cytoskeletal network that contained microtubules, F-actin and associated motor proteins from the Xenopus egg extract (Salman et al., 2005). As native VirE2 molecules are not imported into the nucleus of mammalian cells (reviewed in Lacroix et al., 2006b), an 'animalized' form of VirE2 was used, in which point mutations reconstitute an active nuclear localization signal (NLS) of the widely conserved, bipartite type that is actively transported into the animal cell nucleus. Automated tracking coupled with statistical analysis revealed that 'animalized' VirE2-ssDNA complexes were actively transported along the microtubule (but not actin) network and that this movement was dynein (but not kinesin)-dependent (Salman et al., 2005). Thus, the Agrobacterium T-complex is likely transported through the host cell cytoplasm by a cellular motorassisted mechanism (Salman et al., 2005; Tzfira, 2006). The identity of these motor proteins in plant cells, however, remains obscure. Our recent data (T. Tzfira, unpublished) suggest that a dynein-like Arabidopsis protein (DLC3) may be involved in the intracellular trans- 
port of the Agrobacterium T-complexes, through interaction with another host protein, VIP1, which mediates the recognition of VirE2 by the nuclear import machinery of the plant cell (see below). In this scenario, DLC3, a motorlike protein which associates with the plant microtubule network (T. Tzfira, unpublished) may function as a molecular link between VIP1-VirE2-T-DNA complexes and the microtubule track system. Obviously, further studies are required to examine the role of DLC3, a potential member of a new family of plant molecular motors, in the T-complex movement within plant cells and to identify additional components of plant dynein-based motors likely involved in this transport process.

\section{Nuclear import of the T-complex}

The diameter of the mature T-complex (c. $15 \mathrm{~nm}$ ) (AbuArish et al., 2004) substantially exceeds the diffusion size exclusion limit of the nuclear pore complex (NPC) $(9 \mathrm{~nm})$, indicating that T-complexes enter the cell nucleus by an active mechanism mediated by the nuclear import machinery of the host cell (Fig. 2). Because T-complexes are polar structures, their nuclear import is thought to occur in a polar fashion where the VirD2 molecule attached to the $5^{\prime}$ end of the T-strand may initiate the import process (reviewed in Sheng and Citovsky, 1996). That both VirD2 and VirE2 accumulate in the plant cell nucleus (reviewed in Lacroix et al., 2006b) suggests that not only VirD2, but also VirE2 is involved in the T-complex nuclear import. This concept is supported by the observations that T-DNA expression and/or tumorigenicity is reduced in plants inoculated with Agrobacterium strains expressing either VirD2 with a mutated NLS (e.g. Koukolikova-Nicola et al., 1993; Mysore et al., 1998) or VirE2 with mutations within the protein region required for its nuclear import (Dombek and Ream, 1997). Furthermore, VirE2 alone facilitated nuclear import of fluorescently labelled ssDNA microinjected into living plant cells, and this nuclear import was blocked by a nonhydrolysable GTP analogue, a known specific inhibitor of nuclear import (Zupan et al., 1996). The important role of VirE2 in the T-DNA nuclear import was also demonstrated by a complementation assay, in which a mutated Agrobacterium strain lacking the entire VirE2 and expressing a NLS-mutated VirD2 produced tumours on VirE2expressing transgenic plants, but not on wild-type tobacco plants (Gelvin, 1998).

While a certain functional redundancy between VirE2 and VirD2 during the T-complex nuclear import may exist, the combined action of these Vir proteins may be required for the efficient polar translocation of the T-complex into the host nucleus (Ziemienowicz et al., 2001). Thus, VirD2 and VirE2 likely possess specific functional differences which allow them to perform differ- ent, but complementary functions during nuclear import of the T-complexes. Indeed, while VirD2 was imported into the nucleus of both plant and non-plant cells (e.g. Howard et al., 1992; Ziemienowicz et al., 1999; Rhee et al., 2000), VirE2 targeted to the nuclei of plant cells (e.g. Citovsky et al., 1992; 1994), but not of Xenopus oocytes and Drosophila embryos (Guralnick et al., 1996), human HeLa cells (Tzfira et al., 2001), or yeast cells (Rhee et al., 2000; Tzfira et al., 2001), suggesting that nuclear import of VirD2 and VirE2 in plant cells may occur by different mechanisms. Interestingly, when the nuclear import of VirD2 and VirE2 was studied in permeabilized human cells, both proteins were accumulated to the cell nuclei, but only VirD2, and not VirE2, was able to import ssDNA (Ziemienowicz et al., 1999). In a similar in vitro study using plant cell nuclei, VirD2 was sufficient for the import of short ssDNA, but only the combined function of VirE2 and VirD2 allowed the import of long ssDNA molecules (Ziemienowicz et al., 2001), suggesting that while VirD2 initially directs the T-strand into the NPC, VirE2 may package it in a transferable form and assist translocation of the entire T-complex into the host cell nucleus.

If VirD2 and VirE2 employ different cellular mechanisms for their nuclear entry and perform different functions during transformation, then they are expected to utilize different subsets of cellular factors for their activities. Indeed, VirD2 and VirE2 interact with host factors that belong to distinctly different biological systems of the host cell (Ballas and Citovsky, 1997; Deng et al., 1998; Tzfira et al., 2001; Bakó et al., 2003). For example, VirD2 was shown to interact with a set of plant cyclophilins that include Roc1, Roc2, Roc3, Roc4 and Roc5/CypA (Deng et al., 1998; Bakó et al., 2003). Cyclophilins are a family of conserved peptidyl-prolyl cis-trans isomerases (PPlases) (Tao et al., 2004) which also function as molecular chaperons. Cyclosporin A, known to bind cyclophilins and block their PPlase activity, inhibited the VirD2-CypA interaction in vitro and blocked the Agrobacterium infection of Arabidopsis and tobacco tissues (Deng et al., 1998). While the exact function of CypA and other VirD2interacting cyclophilins in Agrobacterium infection remains unclear, they may act as molecular chaperons that maintain the proper conformation of VirD2 during its transport through the cytoplasm and/or into the nucleus of the host cell (Deng et al., 1998).

Agrobacterium may also use a host regulatory phosphorylation/dephosphorylation machinery to control the nuclear import of VirD2 which is phosphorylated by nuclear cyclin-dependent kinase-activating kinases (CAK2Ms) from alfalfa and Arabidopsis (Bakó et al., 2003). This phosphorylation is likely reversed by a type 2C serine/threonine protein phosphatase, designated DIG3, which interacts with the NLS-containing C-terminal 
region of VirD2 (cVirD2) (Tao et al., 2004). Overexpression of DIG3 in tobacco BY2 protoplasts partially inhibited nuclear import of $\mathrm{cVirD2}$, which led the authors to suggest that DIG3 may negatively regulate nuclear import of T-complexes (Tao et al., 2004).

Directly reflecting its capacity for active nuclear import, VirD2 was found to interact with AtKAP $\alpha$ (Ballas and Citovsky, 1997) (Fig. 2), a member of a conserved family of importin/karyopherin $\alpha$ proteins, known to bind NLS and mediate nuclear import in plant and non-plant cells. The interaction of AtKAP $\alpha$ with VirD2 depended on the presence of the C-terminal bipartite NLS of VirD2. The ability of AtKAP $\alpha$ to potentiate the nuclear import of VirD2 in permeabilized yeast cells, the interaction of VirD2 with three other members of the Arabidopsis importin $\alpha$ family (Bakó et al., 2003), and the observation that an Arabidopsis mutant in one of the importin $\alpha$ genes is resistant to Agrobacterium infection (Zhu et al., 2003b) further support the idea that Agrobacterium utilizes the importin $\alpha$-dependent nuclear import machinery of the host cell for nuclear uptake of the invading T-complexes.

Consistent with its inability to enter the cell nucleus in non-plant systems, VirE2 failed to interact with AtKAP $\alpha$ in the yeast two-hybrid assay (Ballas and Citovsky, 1997). Instead, VirE2 was found to bind the Arabidopsis VIP1, a novel basic-zipper (bZIP) protein (Tzfira et al., 2001). VIP1 formed ternary complexes with VirE2 and ssDNA in vitro, whereas VIP1 and VIP1-VirE2 complexes accumulated in the plant cell nucleus within living plant cells (Tzfira et al., 2001; 2004a; Lacroix et al., 2005; Li et al., 2005a). VIP1 also underwent nuclear import in yeast and mammalian cells, and it promoted nuclear import of VirE2 in these non-plant systems, both in vivo and in permeabilized human cells (Tzfira et al., 2001; Citovsky et al., 2004). Because no close homologues of VIP1 were found in non-plant databases (Tzfira et al., 2001), VIP1 may represent the plant-specific factor responsible for the ability of plant, but not non-plant cells to support nuclear import of VirE2.

The importance of VIP1 for the transformation process was underscored by the observations that antisense suppression of VIP1 rendered the resulting transgenic plants resistant to both transient and stable genetic transformation by Agrobacterium (Tzfira et al., 2001) whereas overexpression of VIP1 increased the susceptibility to transformation (Tzfira et al., 2002). Because VIP1 interacted with plant and animal importins $\alpha$ in the yeast twohybrid assay (Tzfira et al., 2002) and in vitro (Citovsky et al., 2004), and nuclear import of VIP1 in yeast cells absolutely required the presence of importin $\alpha$ (Tzfira et al., 2002), it was suggested that Agrobacterium uses VIP1 as a molecular adaptor between VirE2 and the importin $\alpha$-dependent nuclear import pathway of the host cell (Fig. 2). Indeed, VIP1 assembled in ternary com- plexes with VirE2 and importin $\alpha$ in vitro (Citovsky et al., 2004).

Interestingly, VirE3, an Agrobacterium protein exported to the host plant cells (Lacroix et al., 2005), mimicked the function of VIP1 in the VirE2 nuclear import by interacting with both VirE2 and a plant importin $\alpha$ and promoting nuclear accumulation of VirE2 in mammalian cells and in plants with suppressed expression of VIP1 (Lacroix et al., 2005). These observations may explain how Agrobacterium can genetically transform non-plant cells that lack VIP1 (e.g. yeast and human cells, reviewed in Lacroix et al., 2006a). Thus, although Agrobacterium takes advantage of basic biological systems of the host cell for infection, it also may have evolved to produce a 'backup' system of Vir proteins that are exported into the host cells and augment the cellular functions critical for infection. This idea is consistent with the role of VirE3 as one of the bacterial host range factors (Hirooka and Kado, 1986), which may compensate for the absence or very low levels of VIP1 in some plants, and it may reflect a more general ability of infectious microorganisms to encode and export protein functions, likely acquired by convergent evolution, similar to those normally provided by the host cell (Nagai and Roy, 2003).

\section{Chromatin targeting of the T-complex}

Once inside the nucleus, the T-strand must be delivered to site of its future integration in the host chromatin. While the exact sequence of events that mediate this intranuclear transport and chromatin targeting is still unknown, various plant factors and several molecular mechanisms have been implicated in these concluding steps of the transformation process. Specifically, CAK2M and TATA box-binding protein (TBP) both of which bind VirD2 (Bakó et al., 2003), VIP1 which binds VirE2 (Tzfira et al., 2001) and core histones which bind VIP1 (Li et al., 2005a; Loyter et al., 2005) may function in chromatin targeting of the T-complex. CAK2M interacts with the largest subunit of RNA polymerase II, and the latter recruits TBPs not only for transcription, but also for control of transcriptioncoupled DNA repair. Thus, CAK2M and TBP represent the components of the plant transcriptional and DNA repair machineries, and their interaction with VirD2 (Bakó et al., 2003) may target the latter and its cognate T-strand and/or the entire T-complex to the host chromatin (Fig. 2).

By analogy to many other plant bZIP proteins, VIP1 may also be a component of the cellular transcription machinery; in addition, VIP1 has been implicated in decondensation of the plant chromatin (Avivi et al., 2004). Thus, the association of VIP1 with VirE2 (Tzfira et al., 2001) may promote chromatin targeting of the T-complex (Fig. 2). Indeed, VIP1 was found to interact with all four core histones of Xenopus in vitro (Loyter et al., 2005), and 
with at least one Arabidopsis core histone, $\mathrm{H} 2 \mathrm{~A}$, in planta (Li et al., 2005a; Loyter et al., 2005), and plant H2A is known to be essential for T-DNA integration (Mysore et al., 2000a). The VIP1-H2A interaction was further linked to chromatin targeting by the findings that an insertional Arabidopsis mutant in the VIP1 gene produced a truncated VIP1 protein still capable of supporting nuclear import of VirE2 and transient T-DNA expression, but unable to interact with $\mathrm{H} 2 \mathrm{~A}$ and promote stable expression of the T-DNA ( $\mathrm{Li}$ et al., 2005a). Finally, chromatin targeting of the T-complex may be also facilitated by another VirE2-interacting protein, VIP2 (Tzfira et al., 2000), with a homology to Negative on TATA-less (Not) transcription factors. Consistent with this function and similarly to the truncated VIP1 mutant (Li et al., 2005a), VIP2 is required for stable, but not for transient transformation of plants by Agrobacterium (A. Anand et al., unpublished). Further supporting the thought that Agrobacterium uses the host transcription machinery for delivery of its T-DNA to points of integration in the host chromatin (Fig. 2), high frequency of T-DNA insertion was observed within the regulatory regions of plant genes (e.g. Alonso et al., 2003).

\section{Targeted proteolysis and T-DNA uncoating}

At least partial uncoating of the T-DNA from its escorting proteins (Fig. 2) is necessary for exposing the T-strand to the host DNA repair machinery which will complement it to the double-stranded form and integrate the latter into the host genome. Potentially, this is achieved by the targeted proteolysis machinery of the host cell. The first indication of targeted proteolysis involvement in the transformation process came from the studies of VirF, a bacterial host range factor (e.g. Regensburg-Tuink and Hooykaas, 1993) exported into the host cell (Vergunst et al., 2000). VirF was shown to possess an F-box domain and interact with several members of the ASK protein family of Arabidopsis homologues of the yeast Skp1 protein (Schrammeijer et al., 2001). F-box and Skp1 represent the conserved components of E3 ubiquitin ligases called SCF (Skp1-Cullin-F-box protein) complexes that mediate and specify protein destabilization by targeted proteasomal degradation (reviewed in Ho et al., 2006).

A later study identified VIP1 as one of the cellular substrates for VirF and demonstrated that VirF destabilizes VIP1 and its cognate VirE2 when coexpressed in yeast cells or in planta, and that, in yeast, this destabilization requires the presence of Skp1 (Tzfira et al., 2004a). Because VirE2 represents the major protein component of the T-complex, its targeted proteolysis by the SCF ${ }^{\text {VirF }}$ complexes may represent a mechanism for T-DNA uncoating prior to or during its integration into the host genome (Tzfira et al., 2004a) (Fig. 2). Consistent with this hypoth- esis, both VirF and ASK1 were found to localize to the plant cell nucleus, the cellular compartment in which the T-DNA uncoating is expected to occur; furthermore, early T-DNA expression was specifically inhibited by a proteasomal inhibitor (Tzfira et al., 2004a). Interestingly VirF is required for transformation of some but not all plant species (e.g. Regensburg-Tuink and Hooykaas, 1993), suggesting that its function may be fulfilled by as yet unidentified nuclear-localized cellular F-box protein(s), and that Agrobacterium may harness the host SCF pathways for its own life cycle.

\section{DNA repair and T-DNA integration}

T-DNA integration is the last and perhaps the most hostdependent step of the transformation process (reviewed in Tzfira et al., 2004b). Host factors are required for complementation of the T-strand molecule to doublestranded DNA (dsDNA), for production of DNA breaks in the host genome and for ligation of the T-DNA molecule into these breaks (Fig. 2). While we are still far from complete understanding of the exact steps that lead to the integration of a single-stranded T-DNA molecule into a double-stranded host genome, recent studies have begun revealing key proteins and possible mechanisms which govern the T-DNA integration process. Early models suggested a general mechanism for T-DNA integration that begins with microannealing of the T-DNA left and right borders to the host genome, followed by production of a nick in the host genome, insertion and ligation of the T-strand molecule into the nick and its conversion to a double-stranded form by gap repair (reviewed in Tzfira et al., 2004b). Recent evidence, however, indicates that double-stranded breaks (DSBs) in the host genome and double-stranded T-DNA intermediates play an important role in the integration process. First, induction of DSBs at specific sites in the host DNA by a rare-cutting restriction enzyme results in frequent insertion of T-DNA molecules into these sites (Salomon and Puchta, 1998). Second, incorporating a recognition site for a rare-cutting restriction enzyme not only in the plant genome, but also within the invading T-DNA resulted in frequent insertion of the digested T-DNA molecules into DSBs; because the restriction endonuclease utilized in these experiments cleaves only dsDNA (Jasin, 1996), the T-strands must have been converted to double-stranded molecules prior to their cleavage and integration into genomic DSBs (Chilton and Que, 2003; Tzfira et al., 2003). It is therefore likely that, also in nature, T-DNA integration involves conversion of the T-strands into double-stranded intermediates which are then directed to naturally occurring DSBs in the host genome for integration (Fig. 2).

Yeast cells, which can be genetically transformed by Agrobacterium, yielded much of what we know today 
about the role of host proteins in the T-DNA integration process. Agrobacterium infection of yeast mutants in specific DNA repair genes allowed identification of Ku70, Rad50, Mre11, Xrs2, Lig4 and Sir4 as key proteins in T-DNA integration via non-homologous (illegitimate) recombination (NHR) pathway (van Attikum and Hooykaas, 2003), and demonstrated that Rad51 and Rad52, but not Rad50, Mre11, Xrs2, Lig4 or Ku70 are essential for T-DNA integration by homologous recombination (HR) (van Attikum et al., 2001). Furthermore, Ku70 (van Attikum et al., 2001) and Rad52 (van Attikum and Hooykaas, 2003) were found to be the key determinants for T-DNA integration via HR or NHR respectively, and double mutation of the Ku70 and Rad52 genes resulted in complete blockage of T-DNA integration (van Attikum and Hooykaas, 2003). Another yeast DNA repair protein, Rad54, promoted a high-frequency gene targeting in transgenic plants (Shaked et al., 2005).

In plant cells, T-DNA integration occurs mainly through NHR, even when the T-DNA shares high homology with the host genome, indicating that Agrobacterium may be exclusively using the host non-homologues end-joining (NHEJ) DNA repair machinery during the integration step. Indeed, the critical role of KU80, a key participant of NHEJ which usually functions in a complex with KU70 and DNA protein kinase (reviewed in Tzfira et al., 2004b; Lacroix et al., 2006b) (Fig. 2), during T-DNA integration in Arabidopsis somatic tissues was recently revealed by the observations that Arabidopsis insertional mutants in the $K U 80$ gene are defective in T-DNA integration in somatic cells, and that complexes between KU80 and doublestranded T-DNA molecules can be immunoprecipitated from Agrobacterium-infected plants (Li et al., 2005b). The role of KU80 during the transformation of germ-line cells, however, is less clear as it has been reported to be both required (Friesner and Britt, 2003) and dispensable (Gallego et al., 2003) for T-DNA integration. Similarly, the role of the Arabidopsis LIG4 ligase, another NHEJ participant (Fig. 2), in the transformation process remains controversial; LIG4 was dispensable for T-DNA integration in somatic Arabidopsis cells (van Attikum et al., 2003), but it was essential for T-DNA integration in germ-line cells (Friesner and Britt, 2003). Some of these apparently contradictory results may arise from the nature of the germline transformation (Ye et al., 1999) which was used in many of these studies and performed under relatively uncontrolled conditions. Alternatively, these controversies may reflect fundamental differences between host factors required for T-DNA integration in somatic cells (Zhu et al., 2003b) and in germ-line cells (Ye et al., 1999). Indeed, many other Arabidopsis mutants resistant to Agrobacterium somatic transformation (rat mutants) (Zhu et al., 2003b) remained susceptible to the germ-line transformation (Mysore et al., 2000b).
T-DNA integration may also require the participation of the host DNA-packaging proteins. For example, the Arabidopsis histone $\mathrm{H} 2 \mathrm{~A}$, which displays higher expression levels in tissues more susceptible to Agrobacterium infection (Yi et al., 2002), is essential for T-DNA integration in somatic cells (Mysore et al., 2000a). Thus, in addition or alternatively to its described above role in directing the T-complex molecules to the integration site ( $\mathrm{Li}$ et al., 2005a; Loyter et al., 2005), H2A may be involved in relaxing the host DNA structure (Mysore et al., 2000a). Indeed, also as mentioned above, genome-wide T-DNA insertional analysis revealed a bias for T-DNA integration into intergenic regions which also include promoter sequences and $5^{\prime}$ and $3^{\prime}$ untranslated regions (e.g. Alonso et al., 2003) that are most likely to be temporarily unpacked and may serve as 'hot points' of attraction for T-DNA integration. In addition, because the events of integration and chromatin targeting are temporally and spatially adjacent, at least some of the host factors involved in chromatin targeting (see above) may also facilitate integration, and vice versa.

\section{Plant immunity and T-DNA expression}

Plants perceive Agrobacterium and the transferred transgenes as foreign invaders and use their defence systems to battle the infection process and expression of foreign genes. Recent analyses of the response of different plant species to Agrobacterium infection demonstrated that, in Ageratum plants, a number of general defence-related genes were moderately induced; this induction was dramatically higher when the plants were challenged by an attachment-defective Agrobacterium mutant, suggesting that Agrobacterium binding to the host cell may moderate its defence response (Ditt et al., 2001; 2005). Similarly, the Arabidopsis transcriptome responded to Agrobacterium infection by upregulating numerous general defence genes, and this induction of defence was best observed relatively late (24-48 h) after inoculation (Ditt et al., 2006). In tobacco, however, Agrobacterium infection activated defence response genes early after inoculation (6-12 h), but suppressed them later (24-36 h) in the infection (Veena et al., 2003).

To date, three specific plant immunity pathways have been implicated in the host response to Agrobacterium: perception of bacterial pathogen-associated molecular patterns (PAMPs) which leads to production of reactive oxygen species and reinforcement of cell walls via callose deposition (reviewed in Nürnberger et al., 2004), systemic acquired resistance (SAR) which involves accumulation of salicylic acid (SA) and activation of pathogenesisrelated (PR) genes (reviewed in Durrant and Dong, 2004), and RNA silencing which promotes cleavage/translational inhibition of the target mRNA and epigenetic modification 
of its gene (reviewed in Bisaro, 2006). Recent studies indicate that while Agrobacterium is susceptible to the PAMP perception defence (Zipfel et al., 2006), it has evolved to counteract SAR (Gaspar et al., 2004) and RNA silencing (Dunoyer et al., 2006).

PAMP perception likely reduces the Agrobacteriuminduced genetic transformation because an Arabidopsis mutant in the EFR gene, which encodes a receptor kinase essential for perception of the bacterial EF-Tu PAMP, was super-susceptible to transformation (Zipfel et al., 2006). In contrast, the SAR response of the host plant is likely inhibited by Agrobacterium infection based on the observations that infected Arabidopsis plants exhibited reduction in SA accumulation and PR1 and PR5 gene expression levels which were even lower than their already low levels in healthy plants (Gaspar et al., 2004). These Agrobacterium effects on SAR may involve the host lysine-rich arabinogalactan-protein AtAGP17 because a mutant in the AtAGP17 gene (rat1) retained its $P R 1$ and $P R 5$ expression levels in the presence of Agrobacterium and became resistant to the infection (Gaspar et al., 2004). Besides SAR, Agrobacterium has evolved to counteract the RNA silencing response of the host. Intriguingly, the interrelationship between Agrobacterium infection and RNA silencing is very complex because whereas the development of Agrobacterium-induced tumours required suppression of RNA silencing mediated by short interfering RNAs (siRNAs), it mandated a functional microRNA (miRNA)-mediated silencing because miRNA-deficient plants were almost immune to infection (Dunoyer et al., 2006). Furthermore, the suppression of siRNA-mediated gene silencing occurred only within tumours, but not in the tissues at earlier stages of the transformation process or upon transformation by nononcogenic Agrobacterium (Dunoyer et al., 2006). The silencing suppression in tumours occurred via inhibition of siRNA synthesis, potentially due to interference with Dicer (Dunoyer et al., 2006). The specific factors responsible for suppression of RNA silencing in the Agrobacterium-induced tumours remain unknown. By analogy to plant viruses many of which encode RNA silencing suppressors (reviewed in Bisaro, 2006), Agrobacterium may encode and transfer to plant its own silencing suppressor(s); because the suppression occurs only in tumours, the suppressor may be specified by the oncogenic T-DNA. Alternatively, Agrobacterium may suppress silencing indirectly, via developmental changes induced in the transformed cells by phytohormones. In this regard, it is noteworthy that miRNA pathways are required for cell fate determination (Bartel and Bartel, 2003) which may be involved in tumorigenesis (Dunoyer et al., 2006), and that Agrobacterium virulence is negatively feedback-regulated by phytohormones (Liu and Nester, 2006).

\section{Concluding remarks}

To date, we have come to realize that Agrobacterium not only uses a complex set of its own virulence functions to genetically transform the host cells, but it also subverts many basic cellular processes for this purpose. Furthermore, Agrobacterium is able to actively interfere with some of these systems to escape host defence. The complexity of the eukaryotic systems utilized and/or affected by Agrobacterium is just beginning to emerge. Better understanding of the host biological processes involved in transformation will unravel principles that govern Agrobacterium-host cell interactions which result in the unique event of trans-kingdom gene transfer, afford novel insights into the cellular processes themselves, and help develop new strategies for efficient genetic manipulation of plant and non-plant organisms.

\section{Acknowledgements}

We apologize to colleagues whose works have not been cited due to the lack of space. Our labs are supported by grants from BRDC and the HFSP (to T.T.), and from NIH, NSF, USDA, BARD and BSF (to V.C.).

\section{References}

Abu-Arish, A., Frenkiel-Krispin, D., Fricke, T., Tzfira, T., Citovsky, V., Grayer Wolf, S., and Elbaum, M. (2004) Three-dimensional reconstruction of Agrobacterium VirE2 protein with single-stranded DNA. J Biol Chem 279: 25359-25363.

Alonso, J.M., Stepanova, A.N., Leisse, T.J., Kim, C.J., Chen, H., Shinn, P., et al. (2003) Genome-wide insertional mutagenesis of Arabidopsis thaliana. Science 301: 653657.

van Attikum, H., and Hooykaas, P.J.J. (2003) Genetic requirements for the targeted integration of Agrobacterium T-DNA in Saccharomyces cerevisiae. Nucleic Acids Res 31: 826-832.

van Attikum, H., Bundock, P., and Hooykaas, P.J.J. (2001) Non-homologous end-joining proteins are required for Agrobacterium T-DNA integration. EMBO J 20: 65506558.

van Attikum, H., Bundock, P., Overmeer, R.M., Lee, L.Y., Gelvin, S.B., and Hooykaas, P.J.J. (2003) The Arabidopsis AtLIG4 gene is required for the repair of DNA damage, but not for the integration of Agrobacterium T-DNA. Nucleic Acids Res 31: 4247-4255.

Avivi, Y., Morad, V., Ben-Meir, H., Zhao, J., Kashkush, K., Tzfira, T., et al. (2004) Reorganization of specific chromosomal domains and activation of silent genes in plant cells acquiring pluripotentiality. Dev Dyn 230: 12-22.

Bakó, L., Umeda, M., Tiburcio, A.F., Schell, J., and Koncz, C. (2003) The VirD2 pilot protein of Agrobacterium-transferred DNA interacts with the TATA box-binding protein and a nuclear protein kinase in plants. Proc Natl Acad Sci USA 100: 10108-10113. 
Ballas, N., and Citovsky, V. (1997) Nuclear localization signal binding protein from Arabidopsis mediates nuclear import of Agrobacterium VirD2 protein. Proc Natl Acad Sci USA 94: 10723-10728.

Bartel, B., and Bartel, D.P. (2003) MicroRNAs: at the root of plant development? Plant Physiol 132: 709-717.

Bisaro, D.M. (2006) Silencing suppression by geminivirus proteins. Virology 344: 158-168.

Burr, T.J., Bazzi, C., Sule, S., and Otten, L. (1998) Crown gall of grape: biology of Agrobacterium vitis and the development of disease control strategies. Plant Dis 82: 12881297.

Chilton, M.D., and Que, Q. (2003) Targeted integration of T-DNA into the tobacco genome at double-strand breaks: new insights on the mechanism of T-DNA integration. Plant Physiol 133: 956-965.

Christie, P.J., Atmakuri, K., Krishnamoorthy, V., Jakubowski, S., and Cascales, E. (2005) Biogenesis, architecture, and function of bacterial type IV secretion systems. Annu Rev Microbiol 59: 451-485.

Citovsky, V., Zupan, J., Warnick, D., and Zambryski, P.C. (1992) Nuclear localization of Agrobacterium VirE2 protein in plant cells. Science 256: 1802-1805.

Citovsky, V., Warnick, D., and Zambryski, P.C. (1994) Nuclear import of Agrobacterium VirD2 and VirE2 proteins in maize and tobacco. Proc Natl Acad Sci USA 91: 32103214.

Citovsky, V., Kapelnikov, A., Oliel, S., Zakai, N., Rojas, M.R., Gilbertson, R.L., et al. (2004) Protein interactions involved in nuclear import of the Agrobacterium VirE2 protein in vivo and in vitro. J Biol Chem 279: 29528-29533.

Deng, W., Chen, L., Wood, D.W., Metcalfe, T., Liang, X., Gordon, M.P., et al. (1998) Agrobacterium VirD2 protein interacts with plant host cyclophilins. Proc Natl Acad Sci USA 95: 7040-7045.

Ditt, R.F., Nester, E.W., and Comai, L. (2001) Plant gene expression response to Agrobacterium tumefaciens. Proc Natl Acad Sci USA 98: 10954-10959.

Ditt, R.F., Nester, E., and Comai, L. (2005) The plant cell defense and Agrobacterium tumefaciens. FEMS Microbiol Lett 247: 207-213.

Ditt, R.F., Kerr, K.F., de Figueiredo, P., Delrow, J., Comai, L., and Nester, E.W. (2006) The Arabidopsis thaliana transcriptome in response to Agrobacterium tumefaciens. Mol Plant Microbe Interact 19: 665-681.

Dombek, P., and Ream, L.W. (1997) Functional domains of Agrobacterium tumefaciens single-stranded DNA-binding protein VirE2. J Bacteriol 179: 1165-1173.

Dunoyer, P., Himber, C., and Voinnet, O. (2006) Induction, suppression and requirement of RNA silencing pathways in virulent Agrobacterium tumefaciens infections. Nat Genet 38: 258-262.

Durrant, W.E., and Dong, X. (2004) Systemic acquired resistance. Annu Rev Phytopathol 42: 185-209.

Friesner, J., and Britt, A.B. (2003) Ku80- and DNA ligase IV-deficient plants are sensitive to ionizing radiation and defective in T-DNA integration. Plant J 34: 427-440.

Gallego, M.E., Bleuyard, J.Y., Daoudal-Cotterell, S., Jallut, N., and White, C.I. (2003) Ku80 plays a role in nonhomologous recombination but is not required for T-DNA integration in Arabidopsis. Plant J 35: 557-565.
Gaspar, Y.M., Nam, J., Schultz, C.J., Lee, L.Y., Gilson, P.R., Gelvin, S.B., and Bacic, A. (2004) Characterization of the Arabidopsis lysine-rich arabinogalactan-protein AtAGP17 mutant (rat1) that results in a decreased efficiency of Agrobacterium transformation. Plant Physiol 135: 21622171.

Gelvin, S.B. (1998) Agrobacterium VirE2 proteins can form a complex with $\mathrm{T}$ strands in the plant cytoplasm. $J$ Bacteriol 180: 4300-4302.

Gelvin, S.B. (2003) Agrobacterium-mediated plant transformation: the biology behind the 'gene-jockeying' tool. Microbiol Mol Biol Rev 67: 16-37.

Guralnick, B., Thomsen, G., and Citovsky, V. (1996) Transport of DNA into the nuclei of Xenopus oocytes by a modified VirE2 protein of Agrobacterium. Plant Cell 8: 363-373.

Henry, T., Gorvel, J.P., and Meresse, S. (2006) Molecular motors hijacking by intracellular pathogens. Cell Microbiol 8: 23-32.

Hirooka, T., and Kado, C.I. (1986) Location of the right boundary of the virulence region on Agrobacterium tumefaciens plasmid pTiC58 and a host specifying gene next to the boundary. J Bacteriol 168: 237-243.

Ho, M.S., Tsai, P.I., and Chien, C.T. (2006) F-box proteins: the key to protein degradation. J Biomed Sci 13: 181-191.

Howard, E., Zupan, J., Citovsky, V., and Zambryski, P.C. (1992) The VirD2 protein of A.tumefaciens contains a C-terminal bipartite nuclear localization signal: implications for nuclear uptake of DNA in plant cells. Cell 68: 109-118.

Hwang, H.H., and Gelvin, S.B. (2004) Plant proteins that interact with VirB2, the Agrobacterium tumefaciens pilin protein, mediate plant transformation. Plant Cell 16: 31483167.

Jasin, M. (1996) Genetic manipulation of genomes with rarecutting endonucleases. Trends Genet 12: 224-228.

Kado, C.I. (2000) The role of the T-pilus in horizontal gene transfer and tumorigenesis. Curr Opin Microbiol 3: 643648.

Koukolikova-Nicola, Z., Raineri, D., Stephens, K., Ramos, C., Tinland, B., Nester, E.W., and Hohn, B. (1993) Genetic analysis of the virD operon of Agrobacterium tumefaciens: a search for functions involved in transport of T-DNA into the plant cell nucleus and in T-DNA integration. $J$ Bacteriol 175: 723-731.

Lacroix, B., Vaidya, M., Tzfira, T., and Citovsky, V. (2005) The VirE3 protein of Agrobacterium mimics a host cell function required for plant genetic transformation. EMBO J 24: 428437.

Lacroix, B., Tzfira, T., Vainstein, A., and Citovsky, V. (2006a) A case of promiscuity: Agrobacterium's endless hunt for new partners. Trends Genet 22: 29-37.

Lacroix, B., Li, J., Tzfira, T., and Citovsky, V. (2006b) Will you let me use your nucleus? How Agrobacterium gets its T-DNA expressed in the host plant cell. Can $J$ Physiol Pharmacol 84: 333-345.

Lai, E.M., and Kado, C.I. (2000) The T-pilus of Agrobacterium tumefaciens. Trends Microbiol 8: 361-369.

Li, J., Krichevsky, A., Vaidya, M., Tzfira, T., and Citovsky, V. (2005a) Uncoupling of the functions of the Arabidopsis VIP1 protein in transient and stable plant genetic transformation by Agrobacterium. Proc Natl Acad Sci USA 102: 5733-5738. 
Li, J., Vaidya, M., White, C., Vainstein, A., Citovsky, V., and Tzfira, T. (2005b) Involvement of KU80 in T-DNA integration in plant cells. Proc Natl Acad Sci USA 102: 1923119236.

Liu, P., and Nester, E.W. (2006) Indoleacetic acid, a product of transferred DNA, inhibits vir gene expression and growth of Agrobacterium tumefaciens C58. Proc Natl Acad Sci USA 103: 4658-4662.

Loyter, A., Rosenbluh, J., Zakai, N., Li, J., Kozlovsky, S.V., Tzfira, T., and Citovsky, V. (2005) The plant VirE2 interacting protein 1. A molecular link between the Agrobacterium T-complex and the host cell chromatin? Plant Physiol 138: 1318-1321.

Luby-Phelps, K. (2000) Cytoarchitecture and physical properties of cytoplasm: volume, viscosity, diffusion, intracellular surface area. Int Rev Cytol 192: 189-221.

McCullen, C.A., and Binns, A.N. (2006) Agrobacterium tumefaciens and plant cell interactions and activities required for interkingdom macromolecular transfer. Annu Rev Cell Dev Biol 22: 101-127.

Mysore, K.S., Bassuner, B., Deng, X.B., Darbinian, N.S., Motchoulski, A., Ream, L.W., and Gelvin, S.B. (1998) Role of the Agrobacterium tumefaciens VirD2 protein in T-DNA transfer and integration. Mol Plant Microbe Interact 11: 668-683.

Mysore, K.S., Nam, J., and Gelvin, S.B. (2000a) An Arabidopsis histone H2A mutant is deficient in Agrobacterium T-DNA integration. Proc Natl Acad Sci USA 97: 948953.

Mysore, K.S., Kumar, C.T., and Gelvin, S.B. (2000b) Arabidopsis ecotypes and mutants that are recalcitrant to Agrobacterium root transformation are susceptible to germ-line transformation. Plant $\mathrm{J}$ 21: 9-16.

Nagai, H., and Roy, C.R. (2003) Show me the substrates: modulation of host cell function by type IV secretion systems. Cell Microbiol 5: 373-383.

Nürnberger, T., Brunner, F., Kemmerling, B., and Piater, L. (2004) Innate immunity in plants and animals: striking similarities and obvious differences. Immunol Rev 198: 249266.

Paulsson, M., and Wadstrom, T. (1990) Vitronectin and type-I collagen binding by Staphylococcus aureus and coagulase-negative streptococci. FEMS Microbiol Immunol 65: 55-62.

Regensburg-Tuink, A.J., and Hooykaas, P.J.J. (1993) Transgenic $N$. glauca plants expressing bacterial virulence gene virF are converted into hosts for nopaline strains of A. tumefaciens. Nature 363: 69-71.

Rhee, Y., Gurel, F., Gafni, Y., Dingwall, C., and Citovsky, V. (2000) A genetic system for detection of protein nuclear import and export. Nat Biotechnol 18: 433-437.

Salman, H., Abu-Arish, A., Oliel, S., Loyter, A., Klafter, J., Granek, R., and Elbaum, M. (2005) Nuclear localization signal peptides induce molecular delivery along microtubules. Biophys J 89: 2134-2145.

Salomon, S., and Puchta, H. (1998) Capture of genomic and T-DNA sequences during double-strand break repair in somatic plant cells. EMBO J 17: 6086-6095.

Schrammeijer, B., Risseeuw, E., Pansegrau, W., Regensburg-Tuïnk, T.J.G., Crosby, W.L., and Hooykaas, P.J.J. (2001) Interaction of the virulence protein VirF of
Agrobacterium tumefaciens with plant homologs of the yeast Skp1 protein. Curr Biol 11: 258-262.

Shaked, H., Melamed-Bessudo, C., and Levy, A.A. (2005) High-frequency gene targeting in Arabidopsis plants expressing the yeast RAD54 gene. Proc Natl Acad Sci USA 102: 12265-12269.

Sheng, J., and Citovsky, V. (1996) Agrobacterium-plant cell interaction: have virulence proteins - will travel. Plant Cell 8: $1699-1710$.

Ye, G.N., Stone, D., Pang, S.Z., Creely, W., Gonzalez, K., and Hinchee, M. (1999) Arabidopsis ovule is the target for Agrobacterium in planta vacuum infiltration transformation. Plant J 19: 249-257.

Swart, S., Logman, T.J., Smit, G., Lugtenberg, B.J., and Kijne, J.W. (1994) Purification and partial characterization of a glycoprotein from pea (Pisum sativum) with receptor activity for rhicadhesin, an attachment protein of Rhizobiaceae. Plant Mol Biol 24: 171-183.

Tao, Y., Rao, P.K., Bhattacharjee, S., and Gelvin, S.B. (2004) Expression of plant protein phosphatase $2 \mathrm{C}$ interferes with nuclear import of the Agrobacterium T-complex protein VirD2. Proc Natl Acad Sci USA 101: 5164-5169.

Tzfira, T. (2006) On tracks and locomotives: the long route of DNA to the nucleus. Trends Microbiol 14: 61-63.

Tzfira, T., Rhee, Y., Chen, M.-H., and Citovsky, V. (2000) Nucleic acid transport in plant-microbe interactions: the molecules that walk through the walls. Annu Rev Microbiol 54: 187-219.

Tzfira, T., Vaidya, M., and Citovsky, V. (2001) VIP1, an Arabidopsis protein that interacts with Agrobacterium VirE2, is involved in VirE2 nuclear import and Agrobacterium infectivity. EMBO J 20: 3596-3607.

Tzfira, T., Vaidya, M., and Citovsky, V. (2002) Increasing plant susceptibility to Agrobacterium infection by overexpression of the Arabidopsis VIP1 gene. Proc Natl Acad Sci USA 99: 10435-10440.

Tzfira, T., Frankmen, L., Vaidya, M., and Citovsky, V. (2003) Site-specific integration of Agrobacterium T-DNA via double-stranded intermediates. Plant Physiol 133: 10111023.

Tzfira, T., Vaidya, M., and Citovsky, V. (2004a) Involvement of targeted proteolysis in plant genetic transformation by Agrobacterium. Nature 431: 87-92.

Tzfira, T., Li, J., Lacroix, B., and Citovsky, V. (2004b) Agrobacterium T-DNA integration: molecules and models. Trends Genet 20: 375-383.

Veena, Doerge, R.W., and Gelvin, S.B. (2003) Transfer of T-DNA and Vir proteins to plant cells by Agrobacterium tumefaciens induces expression of host genes involved in mediating transformation and suppresses host defense gene expression. Plant J 35: 219-236.

Vergunst, A.C., Schrammeijer, B., den Dulk-Ras, A., de Vlaam, C.M.T., Regensburg-Tuink, T.J., and Hooykaas, P.J.J. (2000) VirB/D4-dependent protein translocation from Agrobacterium into plant cells. Science 290: 979-982.

Villemont, E., Dubois, F., Sangwan, R.S., Vasseur, G., Bourgeois, Y., and Sangwan-Norreel, B.S. (1997) Role of the host cell cycle in the Agrobacterium-mediated genetic transformation of Petunia: evidence of an S-phase control mechanism for T-DNA transfer. Planta 201: $160-172$. 
Wagner, V.T., and Matthysse, A.G. (1992) Involvement of vitronectin-like protein in attachment of Agrobacterium tumefaciens to carrot suspension culture cells. J Bacteriol 174: 5999-6003.

Yi, H., Mysore, K.S., and Gelvin, S.B. (2002) Expression of the Arabidopsis histone $\mathrm{H} 2 \mathrm{~A}-1$ gene correlates with susceptibility to Agrobacterium transformation. Plant $J$ 32: 285-298.

Zhu, Y., Nam, J., Carpita, N.C., Matthysse, A.G., and Gelvin, S.B. (2003a) Agrobacterium-mediated root transformation is inhibited by mutation of an Arabidopsis cellulose synthase-like gene. Plant Physiol 133: 1000-1010.

Zhu, Y., Nam, J., Humara, J.M., Mysore, K., Lee, L.Y., Cao, $\mathrm{H}$., et al. (2003b) Identification of Arabidopsis rat mutants. Plant Physiol 132: 494-505.
Ziemienowicz, A., Görlich, D., Lanka, E., Hohn, B., and Rossi, L. (1999) Import of DNA into mammalian nuclei by proteins originating from a plant pathogenic bacterium. Proc Natl Acad Sci USA 96: 3729-3733.

Ziemienowicz, A., Merkle, T., Schoumacher, F., Hohn, B., and Rossi, L. (2001) Import of Agrobacterium T-DNA into plant nuclei: two distinct functions of VirD2 and VirE2 proteins. Plant Cell 13: 369-384.

Zipfel, C., Kunze, G., Chinchilla, D., Caniard, A., Jones, J.D., Boller, T., and Felix, G. (2006) Perception of the bacterial PAMP EF-Tu by the receptor EFR restricts Agrobacteriummediated transformation. Cell 125: 749-760.

Zupan, J., Citovsky, V., and Zambryski, P.C. (1996) Agrobacterium VirE2 protein mediates nuclear uptake of ssDNA in plant cells. Proc Natl Acad Sci USA 93: 2392-2397. 